\title{
Prevalence and Risk Factors for Drug-Induced Liver Injury Among Patients With Rheumatic Diseases Treated With Biological Therapy: A Single-Center Experience
}

\author{
K. BRAZDILOVA ${ }^{1}$, T. KOLLER ${ }^{1}$, Z. KILLINGER ${ }^{1}$, J. PAYER ${ }^{1}$ \\ ${ }^{1} 5^{\text {th }}$ Department of Internal Medicine, Faculty of Medicine, Comenius University Bratislava, \\ University Hospital Bratislava, Bratislava, Slovak Republic
}

Received May 27, 2019

Accepted September 23, 2019

\begin{abstract}
Summary
Drug-induced liver injury (DILI) is a common event in patients with rheumatic diseases (RD) on biological therapy (BT). We aimed at evaluating the prevalence and pattern of DILI. Consecutive RD patients treated with BT were followed for 6 months. ALT and ALP >the upper limit normal (ULN) and <to 3XULN defined injury Grade 1 and $>3 X U L N$ injury Grade 2 . 582 liver function tests (LFTs) in 199 patients were evaluated, median age 53y, $59.3 \%$ females, RA in 108, AS 49, and PSA 42 patients. ALT Grade 1 elevation was observed in $25.6 \%$, transient in $18.6 \%$, persisting in $7 \%$, Grade 2 in $1.5 \%$, ALP Grade 1 in $3.5 \%$, transient in $2 \%$, persisting in $1.5 \%$. We report no case of ALP Grade 2 or Hy's law (ALT>3xULN, bilirubin>2xULN). Patients with persisting ALT elevation had higher BMI (28.23 vs. 25.74, $\mathrm{p}=0.016$ ), lower DAS28 (2.22 vs. $5.28, \mathrm{p}=0.046)$. ALT Grade 1 injury was more frequent with solo tocilizumab compared with other agents $(27.5 \%$ vs. $13.6 \%$, $\mathrm{p}=0.01$ ). DILI was frequent, in $18.6 \%$ transient, in $7 \%$ persisting, Grade 2 in $1.5 \%$, led to treatment alteration in $0.5 \%$, with higher prevalence on solo tocilizumab therapy. We report no new safety signals for $B T$ in RD.
\end{abstract}

\section{Key words}

Drug-induced liver injury - Rheumatic diseases • Biological therapy

\section{Corresponding author}

T. Koller, 5th Department of Internal Medicine, Faculty of Medicine, Comenius University, University Hospital Bratislava, Ruzinovska 6, 82606 Bratislava, Slovak Republic. E-mail: koller.tomas@gmail.com

\section{Introduction and Aims}

Drug-induced liver injury (DILI) is one of the most common and serious adverse drug reactions (Leise et al. 2014). It is the most common cause of acute liver failure in the USA and Europe and also a frequent differential diagnosis in patients with acute liver injury (Björnsson 2016). Liver injury has been reported from nearly all classes of prescription drugs including small chemical molecules, biological agents, but also traditional Chinese medicines, natural medicines, and herbal and dietary supplements. Nonsteroidal anti-inflammatory drugs (NSAIDs) and antirheumatic agents are among the more frequent causes of hepatotoxic injury (Björnsson 2015). Rheumatic diseases usually require long-term therapy with a single drug, but multiple agents are often required for disease control. Recently, medical therapy for rheumatic diseases is rapidly evolving. New molecules with different mechanisms of action are being developed and marketed. With the widespread use of these new products, clinicians caring for patients with rheumatic disease are challenged with their potential sideeffects and toxicities (Anelli et al. 2012). Rare cases of DILI are usually not captured in clinical trials and occur only after the drug is used in real-life (Kaplowitz 2013). Therefore, the aim of our study was to evaluate liver injury in the cohort of real-life rheumatic disease patients treated with various biological agents as well as with traditional anti-rheumatic drugs. We aimed at evaluating the prevalence of liver injury, the pattern and risk factors as well as the evolution and clinical impact of potential toxicity. 


\section{Patients and Methods}

We included all consecutive patients from the Center for the biological treatment of rheumatic diseases of the teaching University Hospital during the period from $1^{\text {st }}$ January 2015 to $1^{\text {st }}$ June 2016. The inclusion criteria were 1) treatment with biological therapy for rheumatoid arthritis, ankylosing spondylitis or psoriatic arthritis at the center, 2) compliance with regular followup controls during the study period that included blood testing for LFTs at 3-month intervals. We included all eligible patients regardless of the treatment duration. Prior to initiation of the biologic therapy, all patients underwent evaluation for possible liver injury including viral hepatitis (negative HBs antigen, negative anti-HCV) and liver function tests (LFTs). LFTs had to be in the normal range prior to treatment start. Patients who were diagnosed with chronic LFTs elevation or chronic liver disease, patients with severe systemic disease such as chronic heart failure, chronic renal failure, pulmonary hypertension, obstructive pulmonary disease, systemic vasculitis, lupus erythematosus or active malignancies were excluded. Obesity or diabetes was not considered an exclusion criterion. Liver ultrasound or elastography was not systematically performed in all included patients.

Liver injury was monitored by measuring serum aminotransferase activities, alanine aminotransferase (ALT, hepatocellular injury), alkaline phosphatase (ALP, cholestatic injury), gamma glutamyl-transferase (GGT) and bilirubin. Mild liver injury (Grade 1) was defined as ALT, ALP or GGT elevation above the upper limit of the normal range (ULN) and inferior to three times the upper limit of normal (ULN). More severe injury (Grade 2) was defined as ALT elevation above 3 times the ULN, as defined by the Food and drug administration (FDA) or the European medicines agency (EMA). The evolution of liver injury was labeled as transient when ALT elevation was observed on 1 or 2 measurements. Persisting (chronic) elevation was diagnosed when ALT elevation was observed in all three measurements. The severity of the liver injury was also assessed by the prevalence of Hy's law defined by ALT elevation of more than 3 times the ULN and bilirubin elevation superior to two times the ULN. The presence of Hy's law signals the possibility of severe liver injury, acute liver failure or death (Kaplowitz 2013). We also recorded anthropometric variables, disease-specific variables such as activity index (Disease Activity Score, DAS28) and (Bath Ankylosing Spondylitis Disease Activity Index, BASDAI score) and all ongoing therapies among anti-rheumatic drugs and biological agents. The DAS 28 is a system developed and validated by the EULAR (European League Against Rheumatism) to measure the progress and improvement of rheumatoid arthritis, but it's used also in patients with psoriatic arthritis. It's a composite score derived from 4 of these measurements: the number of swollen joints, the number of tender joints, ESR (erythrocyte sedimentation rate) or CRP ( $\mathrm{C}$ reactive protein), and a patient's global assessment of health. The BASDAI is a validated diagnostic test that allows determining the effectiveness of current drug therapy in patients with ankylosing spondylitis. It consists of a 0-10 scale measuring discomfort, pain, and fatigue.

Table 1. Summary statistics and characteristics of 199 patients with rheumatic diseases treated with biological therapy

\begin{tabular}{|c|c|}
\hline & Median [IQR]/n (\%) \\
\hline Age & $53.00[24.00,85.00]$ \\
\hline Males (\%) & $81(40.7)$ \\
\hline Females (\%) & $118(59.3)$ \\
\hline BMI & $26.03[15.44,42.52]$ \\
\hline \multicolumn{2}{|l|}{ Ultrasound $(n=24)$} \\
\hline Disease duration (years) & $12.50[2.00,46.00]$ \\
\hline Rheumatoid arthritis (\%) & $108(54.3)$ \\
\hline DAS 28 score & $2.57[0.63,7.50]$ \\
\hline Ankylosing spondylitis (\%) & $49(24.6)$ \\
\hline BASDAI score & $1.40[0.00,8.90]$ \\
\hline Psoriatic arthritis (\%) & $42(21.1)$ \\
\hline \multicolumn{2}{|l|}{ Ongoing treatment: } \\
\hline Steroids & $38(19.1)$ \\
\hline \multicolumn{2}{|l|}{ Non-steroidal anti- } \\
\hline inflammatory & $27(13.6)$ \\
\hline Methotrexate & $87(43.7)$ \\
\hline Infliximab & $2(1.0)$ \\
\hline Adalimumab & $82(41.2)$ \\
\hline \multicolumn{2}{|l|}{ Adalimumab and } \\
\hline methotrexate & $40(20.1)$ \\
\hline Certolizumab & $4(2.0)$ \\
\hline Etanercept & $34(17.1)$ \\
\hline \multicolumn{2}{|l|}{ Etanercept and } \\
\hline methotrexate & $12(6.0)$ \\
\hline Golimumab & $40(20.1)$ \\
\hline \multicolumn{2}{|l|}{ Golimumab and } \\
\hline methotrexate & $21(10.6)$ \\
\hline Tocilizumab & $32(16.1)$ \\
\hline \multicolumn{2}{|l|}{ Tocilizumab and } \\
\hline methotrexate & $9(4.5)$ \\
\hline
\end{tabular}


Data were assessed by the statistical software package MedCalc v.15.2, Belgium. Numerical variables were expressed as medians and interquartile range and proportions as numbers of cases and percentages. Liver enzyme activities were expressed as multiples of an upper limit of the normal range (xULN). Results were considered significant when $p$ values were inferior to 0.05 .

The study had an observational character with no protocol intervention and it was carried out in accordance with the proceedings of the Helsinki declaration. Data were anonymized according to our institutional guidelines and the study protocol was approved by our institutional ethics committee.

Table 2. Prevalence of all patterns of liver injury during 6 months of follow-up among 199 patients with rheumatic diseases treated with biological therapy

\begin{tabular}{lcc}
\hline & & $\mathbf{N}(\%)$ \\
\hline ALT above ULN (\%) & never & $148(74.4)$ \\
& once & $22(11.1)$ \\
& twice & $15(7.5)$ \\
Transient ALT above ULN (\%) & & $37(18.6)$ \\
Persiting ALT above ULN (\%) & & $14(7.0)$ \\
ALT above $3 x U L N(\%)$ & & $3(1.5)$ \\
ALP above ULN (\%) & never & $192(96.5)$ \\
& once & $2(1.0)$ \\
Transient ALP above ULN (\%) & & $2(1.0)$ \\
Persiting ALP above ULN (\%) & & $3(2.0)$ \\
ALP above $3 x U L N(\%)$ & & $0(0)$ \\
GGT above ULN (\%) & never & $159(79.9)$ \\
& once & $9(4.5)$ \\
Transient GGT above ULN (\%) & & $23(11.6)$ \\
Persiting GGT above ULN (\%) & & $17(8.5)$ \\
GGT above $3 x U L N(\%)$ & & $8(4.0)$ \\
\hline & &
\end{tabular}

ULN - upper limit of normal, ALT - alanine aminotransferase, ALP - alkaline phosphatase, GGT - gamma glutamyl transpeptidase

\section{Results}

During the inclusion period, we included 199 patients with rheumatic diseases and during followup, we evaluated 582 aminotransferase measurements. Summary statistics and characteristics of the study cohort are displayed in Table 1. The median age was 53 years and $59.3 \%$ of patients were females. The median disease duration was 12.5 years. 108 patients had rheumatoid arthritis (54.3\%), 49 had ankylosing spondylitis (24.6\%) and 42 had psoriatic arthritis (21.1\%). All patients were treated with biological therapy. In addition, 87 patients were treated with methotrexate $(43.7 \%), 27$ patients with non-steroidal anti-inflammatory agents (13.6\%) and 38 patients with steroids (19.1\%).

The prevalence of liver injury in our study cohort is displayed in Table 2. ALT elevation above the ULN was observed in 51 patients (25.6\%). Mild transient ALT elevation was observed in 37 patients (18.6\%) and persisting (chronic) ALT elevation in 14 patients $(7 \%)$. ALT elevation above 3 times the ULN (Grade 2) was observed in 3 cases $(1.5 \%)$. One patient was treated with solo golimumab, one patient with golimumab and methotrexate (transient elevations) and one patient with etanercept and methotrexate (persisting elevation). In this late patient, the treatment with etanercept has been stopped leading to normalization of liver test abnormalities. ALP elevation was observed in 7 patients $(3.5 \%)$ and it was transient in 4 cases $(2 \%)$ and persisting in 3 cases $(1.5 \%)$. ALP elevation above three times the ULN has not been observed. GGT elevation above ULN was observed in 40 patients $(20.1 \%)$, it was transient in 23 cases $(11.6 \%)$ and persisting in 17 cases $(8.5 \%)$. GGT elevation above 3 times the ULN was observed in 8 patients ( $4 \%$ ).

Comparison of recorded variables between 14 patients with persisting ALT elevation above ULN and the remaining study cohort is displayed in Table 3. We found that patients with elevated ALT had higher BMI (median 28.23 vs. 25.74, $\mathrm{p}=0.016$ ), lower DAS28 score (median 2.22 vs. 5.28, $\mathrm{p}=0.046$ ) and there was a trend towards more frequent treatment with tocilizumab (35.7 \% vs. $14.6 \%, p=0.054)$.

Finally, we analyzed the prevalence of three most clinically relevant liver injury patterns (ALP above ULN, ALT above ULN, ALT above 3xULN) in Table 4 for six different biological agents used in our cohort: adalimumab, certolizumab, etanercept, golimumab, infliximab, and tocilizumab. We observed that the prevalence of ALT elevation above the ULN was more frequent among patients treated with tocilizumab (25\%) compared with other agents used in our cohort (13.6\%, $\mathrm{p}=0.01$ ). In addition, by separately analyzing patients on solo tocilizumab therapy ( $n=23,69$ ALT measurements) and tocilizumab combined with methotrexate $(n=9$, 27 ALT measurements), only patients treated with tocilizumab alone had more frequent mild ALT elevations. 
Table 3. Comparison of patients with and without persisting ALT elevation (6 months) among 199 patients on biological therapy

\begin{tabular}{lccc}
\hline & no persiting ALT elevation & persisting ALT elevation & P-value \\
& $\mathbf{n = 1 8 5}$ & $\mathbf{n}$ & \\
\hline Age & $53.00[24.00,85.00]$ & $58.50[27.00,77.00]$ & 0.705 \\
Males (\%) & $73(39.5)$ & $8(57.1)$ & 0.26 \\
Females (\%) & $112(60.5)$ & $6(42.9)$ & \\
Body mass index & $25.74[15.44,42.52]$ & $28.23[26.54,38.97]$ & 0.016 \\
Disease duration & $13.00[2.00,46.00]$ & $10.00[6.00,31.00]$ & 0.851 \\
Reumathoid arthritis & $101(54.6)$ & $7(50.0)$ & 0.786 \\
$\quad$ DAS.28 score & $2.58[0.63,7.50]$ & $2.22[0.77,3.13]$ & 0.046 \\
Ankylosing spondylitis & $47(25.4)$ & $2(14.3)$ & 0.524 \\
$\quad$ BASDAI score & $1.41[0.00,8.90]$ & $1.15[1.00,1.30]$ & 0.544 \\
Psoriatic arthritis & $37(20.0)$ & $5(35.7)$ & 0.179 \\
Ongoing treatment: & & & \\
$\quad$ Steroids & $35(18.9)$ & $3(21.4)$ & 0.733 \\
Non steroidal anti-inflammatory drugs & $27(14.6)$ & $0(0.0)$ & 0.224 \\
Metothrexate & $80(43.2)$ & $7(50.0)$ & 0.781 \\
$\quad$ Infliximab & $2(1.1)$ & $0(0.0)$ & 1 \\
Adalimumab & $76(41.1)$ & $6(42.9)$ & 1 \\
Adalimumab and metothrexate & & & \\
Certolizumab & $4(2.2)$ & $0(0.0)$ & 1 \\
Etanercept & $32(17.3)$ & $2(14.3)$ & 0.77 \\
Etanercept and metothrexate & $10(5.4)$ & $2(14.3)$ & 0.18 \\
Golimumab & $40(21.6)$ & $0(0.0)$ & 0.077 \\
Golimumab and metothrexate & $21(11.4)$ & $0(0.0)$ & 0.18 \\
Tocilizumab & $27(14.6)$ & $5(35.7)$ & 0.054 \\
Tocilizumab and metothrexate & $8(4.3)$ & $1(7.1)$ & 0.62 \\
\hline
\end{tabular}

Table 4. Prevalence of liver injury according to different biological therapies among 582 ALT and ALP measurements in 199 patients with rheumatic diseases

\begin{tabular}{|c|c|c|c|c|c|c|c|c|c|}
\hline & & ADA & CZP & ETN & GOL & IFX & TOC & TOC alone & TOC and MTX \\
\hline \multirow{5}{*}{$\begin{array}{l}\text { No. } \\
A L P>U L N \\
(\%)\end{array}$} & & 246 & 12 & 102 & 120 & 6 & 96 & 69 & 27 \\
\hline & no & 243 & 12 & 99 & 115 & 6 & 92 & 65 & 27 \\
\hline & & $(98.8)$ & $(100.0)$ & $(97.1)$ & $(95.8)$ & $(100.0)$ & $(95.8)$ & $(94.2)$ & $(100)$ \\
\hline & yes & 3 & 0 & 3 & 5 & 0 & 4 & 4 & 0 \\
\hline & & $(1.2)$ & $(0.0)$ & $(2.9)$ & $(4.2)$ & $(0.0)$ & $(4.2)$ & $(5.8)$ & (0) \\
\hline \multirow{4}{*}{$\begin{array}{l}A L T>U L N \\
(\%)\end{array}$} & no & 210 & 11 & 85 & 108 & 6 & 72 & 50 & 22 \\
\hline & & $(85.4)$ & (91.7) & $(83.3)$ & $(90.0)$ & $(100.0)$ & $(75.0)$ & $(72.5)$ & $(81.5)$ \\
\hline & yes & 36 & 1 & 17 & 12 & 0 & 24 & 19 & 5 \\
\hline & & (14.6) & $(8.3)$ & (16.7) & $(10.0)$ & $(0.0)$ & $(25.0)^{*}$ & $(27.5)^{*}$ & (18.5) \\
\hline \multirow{4}{*}{$\begin{array}{l}A L T>3 x U L N \\
(\%)\end{array}$} & no & 246 & 12 & 101 & 118 & 6 & 96 & 69 & 27 \\
\hline & & (100.0) & $(100.0)$ & $(99.0)$ & (98.3) & $(100.0)$ & (100.0) & $(100)$ & $(100)$ \\
\hline & yes & 0 & 0 & 1 & 2 & 0 & 0 & 0 & 0 \\
\hline & & $(0.0)$ & $(0.0)$ & $(1.0)$ & $(1.7)$ & $(0.0)$ & $(0.0)$ & $(0)$ & $(0)$ \\
\hline
\end{tabular}

${ }^{*} \mathrm{p}<0.01$, ADA-adalimumab, CZP-certolizumab pegol, ETN-etanercept, GOL-golimumab, IFX-infliximab, TOC-tocilizumab 


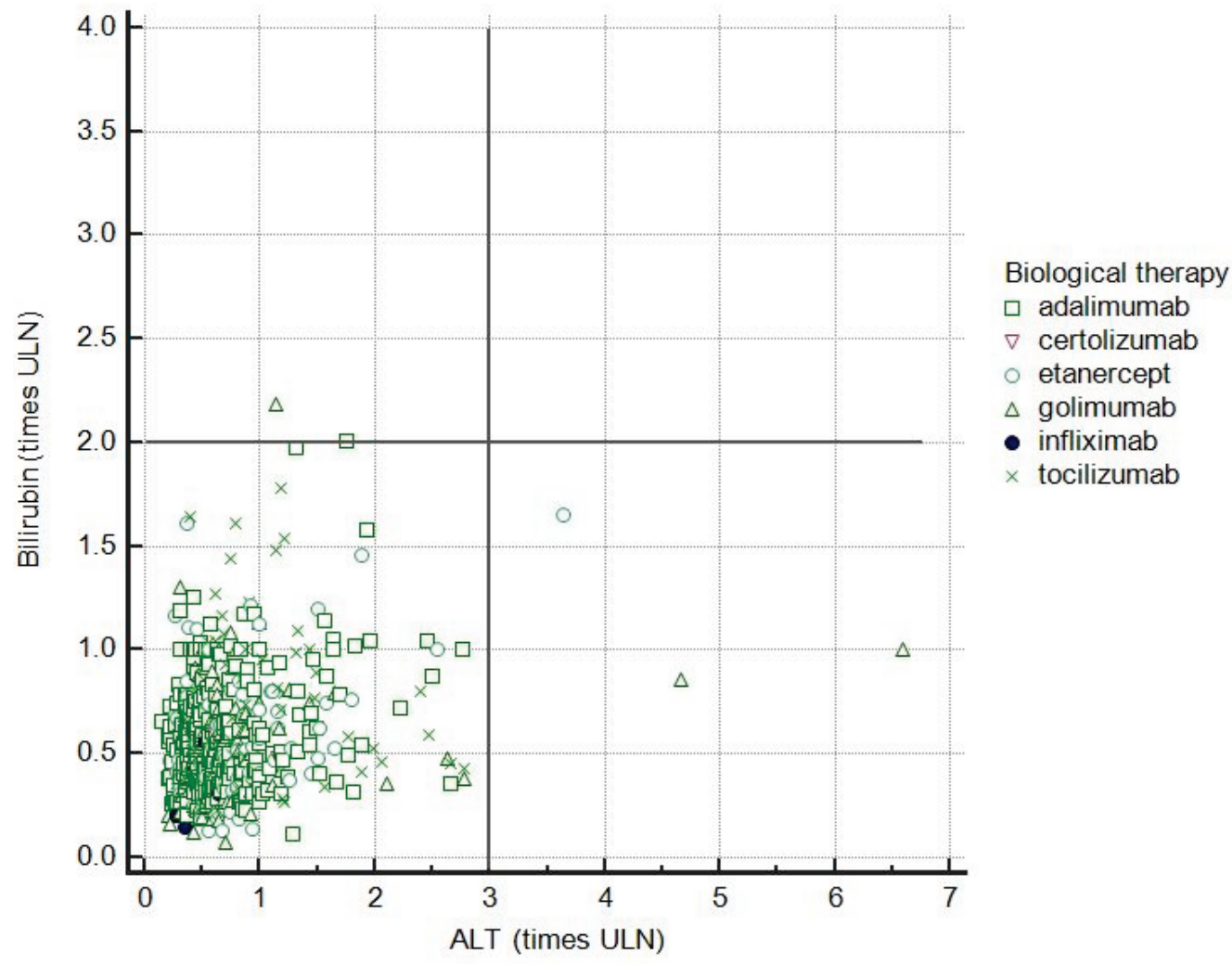

Fig. 1. Plot for $A L T$ and Bilirubin (eDISH) reflecting the prevalence of liver injury among 199 rheumatic patients on biological therapy
During our study, the definition of Hy's law has not been observed in any patient. ALT and bilirubin plot for all measurements in our study is displayed in Fig. 1. illustrating the observed patterns of liver injury.

\section{Discussion}

In our study, we evaluated liver injury in patients with rheumatic diseases treated with various biological therapies for a period of six months. The transient hepatocellular injury was observed in $18.6 \%$ of cases, and persisting injury occurred in $7 \%$ of cases. The cholestatic liver injury occurred transiently in $2 \%$ of cases and persisted in $1.5 \%$ of patients. Patients with persisting mild hepatocellular injury had a higher body mass index and lower DAS28. Therapy with solo tocilizumab compared with other biological agents more frequently caused a mild transient hepatocellular injury. The more severe hepatocellular injury occurred in 3 cases over 6 months $(1.5 \%)$ and led to discontinuation of etanercept in one patient with a favorable outcome.

In the clinical practice of biological therapy, potential side effect and toxicity occur frequently (Sokolove et al. 2010). Our study confirms that aminotransferase abnormalities are found more frequently in patients treated with biologics than in the general population (Liangpunsakul and Chalasani 2005). This particular group of therapeutic agents is known for inducing various types of autoimmune reactions in multiple organ systems (Wood and Caplan 2018). When confronted with the finding of mild liver injury, clinicians face a challenge. On one hand, one can assume that the liver injury is mostly mild and self-limited. It is frequently explained by causes similar to the general population such as liver steatosis (Koller et al. 2017) or non-hepatic origin and is unlikely caused by the administered therapy. However, newly introduced therapies with immunogenic potential do cause immunemediated liver injury. This type of injury is frequently followed by the phase of immune tolerance with aminotransferase activities returning to normal levels (Kaplowitz 2013). On the other hand, any severe liver injury is often accompanied by a slow, but progressive rise in aminotransferase activities. Therefore, when following patients treated with biological therapy, there is still a need for serial aminotransferase monitoring for possible hepatotoxicity (Suk and Kim 2012). Markers predicting the immune-mediated liver injury are yet to come to clinical practice (Farghali et al. 2016). Any abnormalities persisting for more than 3 months or any progressive increase in aminotransferase levels above three times the ULN should alert the clinician to investigate the underlying cause and question the safety of the ongoing treatment. The finding, that patients with 
persisting ALT elevation had lower disease activity scores appears independent on the BMI (analysis not shown). We might speculate for the reasons, however, low prevalence of the severe active RA and the apparent difference in group numbers might allow for a type 1 statistical error.

Biologics such as infliximab, have been reported as one of the most frequent hepatotoxins in the population study from Iceland and the molecule has been implicated in more than one hundred cases of liver injury (Bjornsson et al. 2013). Another frequently used agent adalimumab has also been reported as causing DILI with or without the typical signs of autoimmune hepatitis (Shelton et al. 2015). In clinical studies with golimumab therapy, liver enzyme elevations have been reported more frequent, but no severe cases of liver injury have been described (National Institutes of Health). Two of our three cases of more severe hepatocellular injury were treated with golimumab, but both were transient and due to small numbers of cases this finding cannot be interpreted as a signal for potential hepatotoxicity. Mild and transient liver test elevations have been reported in patients treated with etanercept, but they were less frequent than in infliximab-treated cases. In rare cases, etanercept has been reported causing clinically apparent autoimmune-like hepatitis syndrome (Girolomoni et al. 2012; National Institutes of Health). A single patient from our study cohort had persisting ALT elevation on treatment with etanercept and methotrexate and the clinician has decided to stop therapy due to liver injury as well as the secondary loss of response. Liver tests normalized after stopping the treatment and the patient is now treated with a different drug. Solo tocilizumab therapy in our study has been associated with more frequent aminotransferase elevations compared with other biological molecules. Of note, in patients treated with combination therapy with tocilizumab and methotrexate, the prevalence of liver injury was not different from other agents. We have recently reported a similar observation among patients with inflammatory bowel disease, that patients treated with solo immunosuppressive therapy with infliximab had the highest risk of liver injury (Koller et al. 2017). Thus, it appears that more severe immune suppression could hamper the development of immunemediated liver injury. Clinical trials using tocilizumab have reported more frequent liver test abnormalities that have been mostly mild and transient (Genovese et al. 2013). However, there are some reports in the literature of a severe hepatocellular injury with one fatal case associated with this agent. Of note, clinicians in our center are monitoring liver test abnormalities in patients treated with tocilizumab and in cases of detected liver injury they are lowering the treatment dose (Genovese et al. 2017). Therefore, this strategy may explain our finding, that tocilizumab therapy caused only transient liver test abnormalities in all cases.

Our study has several limitations. First, to our study we included only cases with rheumatic diseases that were treated with biological therapy. Therefore, within our cohort of patients, we did not have a reference group to which we could compare the prevalence of liver injury. Second, we evaluated liver injury only during the six months of follow up, which could have hampered the detection of less frequent events. Third, we evaluated the prevalence of liver injury regardless of the treatment duration. It is known from previous studies, that liver injury tends to occur most frequently during the first three months after treatment initiation. In patients with a documented transient liver injury, we have no data on alcohol consumption or on the presence of fat in the liver during an ultrasound examination. These limitations could have slightly underestimated the true prevalence of liver injury. We might assume that liver steatosis could have explained the liver injury in patients with higher BMI in the group of persisting ALT elevation. Nevertheless, our reported prevalence is within the range that has been reported in clinical trials. Finally, other causes of liver injury in patients treated with immunosuppressive therapy such as reactivation of viral infections (CMV, VZV, etc.) could have been involved.

\section{Conclusion}

In a real-life cohort of 199 patients with rheumatic diseases treated with various biological therapies for 6 months, liver injury was frequent. Its duration was usually transient $(18.6 \%)$ and less frequently persisting ( $7 \%$ ), the severity rarely exceeding values above three times the ULN (1.5\%). The documented liver injury led to treatment alteration in only one patient $(0.5 \%)$. Among risk factors for persisting liver injury were higher body mass index and lower RA disease activity score (DAS28). Patients treated with solo tocilizumab had a higher chance of liver injury compared with another treatment regimen. Our study does not bring new safety signals for biological therapy, but it confirms the need for regular monitoring of liver function tests in these patients.

\section{Conflict of Interest}

There is no conflict of interest. 


\section{References}

ANELLI MG, SCIOSCIA C, GRATTAGLIANO I, LAPADULA G: Old and new antirheumatic drugs and the risk of hepatotoxicity. Ther Drug Monit 34: 622-628, 2012.

BJÖRNSSON ES: Hepatotoxicity by drugs: the most common implicated agents. Int J Mol Sci 17: 224, 2016.

BJÖRNSSON ES: Drug-induced liver injury: an overview over the most critical compounds. Arch Toxicol 89: 327-334, 2015.

BJÖRNSSON ES, BERGMANN OM, BJÖRNSSON HK, KVARAN RB, OLAFSSON S: Incidence, presentation, and outcomes in patients with drug-induced liver injury in the general population of Iceland. Gastroenterol 144: 1419-1425, 2013.

FARGHALI H, KGALALELO KEMELO M, WOJNAROVÁ L, KUTINOVÁ CANOVÁ N: In vitro and in vivo experimental hepatotoxic models in liver research: applications to the assessment of potential hepatoprotective drugs. Physiol Res 65 (Suppl. 4): S417-S425, 2016.

GENOVESE MC, KREMER JM, VAN VOLLENHOVEN RF, ALTEN R, SCALI JJ, KELMAN A, DIMONACO S, BROCKWELL L: Transaminase levels and hepatic events during tocilizumab treatment: pooled analysis of long-term clinical trial safety data in rheumatoid arthritis. Arthritis Rheumatol 69: 1751-1761, 2017.

GENOVESE MC, RUBBERT-ROTH A, SMOLEN JS, KREMER J, KHRAISHI M, GOMEZ-REINO J, SEBBA A, PILSON R, WILLIAMS S, VAN VOLLENHOVEN R: Longterm safety and efficacy of tocilizumab in patients with rheumatoid arthritis: a cumulative analysis of up to 4.6 years of exposure. J Rheumatol 40: 768-780, 2013.

GIROLOMONI G, ALTOMARE G, AYALA F, BERARDESCA E, CALZAVARA-PINTON P, CHIMENTI S, PESERICO A, PUGLISI GUERRA A, VENA GA: Safety of anti-TNF $\alpha$ agents in the treatment of psoriasis and psoriatic arthritis. Immunopharmacol Immunotoxicol 34: 548-60, 2012.

KAPLOWITZ N: Drug-induced liver injury. In: Drug-Induced Liver Disease. N KAPLOWITZ, L DELEVE (eds), Elsevier, 2013, pp 3-14.

KOLLER T, GALAMBOSOVA M, FILAKOVSKA S, KUBINCOVA M, HLAVATY T, TOTH J, KRAJCOVICOVA A, PAYER J: Drug-induced liver injury in inflammatory bowel disease: 1-year prospective observational study. World J Gastroenterol 23: 4102-4111, 2017.

LEISE MD, POTERUCHA JJ, TALWALKAR JA: Drug-induced liver injury. Mayo Clin Proc 89: 95-106, 2014.

LIANGPUNSAKUL S, CHALASANI N: Unexplained elevations in alanine aminotransferase in individuals with the metabolic syndrome: results from the third National Health and Nutrition Survey (NHANES III). Am J Med Sci 329: 111-116, 2005.

NATIONAL INSTITUTES OF HEALTH (n.d.-a) Etanercept, Retrieved 19 April 2019 from https://livertox.nlm.nih.gov/Etanercept.htm.

NATIONAL INSTITUTES OF HEALTH (n.d.-b) Golimumab, Retrieved 19 April 2019 from https://livertox.nlm.nih.gov/Golimumab.htm.

SHELTON E, CHAUDREY K, SAUK J, KHALILI H, MASIA R, NGUYEN DD, YAJNIK V, ANANTHAKRISHNAN AN: New onset idiosyncratic liver enzyme elevations with biological therapy in inflammatory bowel disease. Aliment Pharmacol Ther 41: 972-979, 2015.

SOKOLOVE J, STRAND V, GREENBERG JD, CURTIS JR, KAVANAUGH A, KREMER JM, ANOFREI A, REED G, CALABRESE L, HOOPER M, BAUMGARTNER S, FURST DE: Risk of elevated liver enzymes associated with TNF inhibitor utilisation in patients with rheumatoid arthritis. Ann Rheum Dis 69: 1612-1617, 2010.

SUK KT, KIM DJ: Drug-induced liver injury: present and future. Clin Mol Hepatol 18: 249-257, 2012.

WOOD PR, CAPLAN L: Drug-induced gastrointestinal and hepatic disease associated with biologics and nonbiologic disease-modifying antirheumatic drugs. Rheum Dis Clin North Am 44: 29-43, 2018. 